\title{
ANALES GALDOSIANOS
}

Director - R. Cardona

Año II

1967

Sumario: Denah Lida, 'Sobre el 'Krausismo' de Galdós". Peter G. Earle, "Torquemada: hombre-masa". Antonio Sánchez Barbudo, "Torquemada y la muerte". Frank P. Bowman, "On the Definition of Jesus in Modern Fiction". Ciriaco Morón Arroyo, "Nazarín y Halma: sentido y forma". A. A. Parker, "Nazarin, or the Passion of Our Lord Jesus Christ According to Galdós". Robert H. Russell, "The Christ Figure in Misericordia" (Monograph). Otis H. Green, "Two Deaths: Don Quijote and Marianela". Walter T. Pattison, "El amigo Manso and el amigo Galdós". Vera Colin, "A Note on Tolstoy and Galdós", Rafael Bosch, "Galdós y La teoría de la novela de Lukács". Harold Boudreau, "Bibliographie Essay".

Número "extra" $\$ 6.00$

Pedidos a:

I6I 7 C. L.

University of Pittsburgh

Pittsburgh, Pennsylvania

I5213, U.S.A. 
The Instituto Internacional de Literatura Iberoamericana was organized in 1938 in order to advance the study of Iberoamerican Literature and to promote cultural relations among the peoples of the Americas.

To this end, the Institute publishes the Revista Iberoamericana twice a year and sponsors the publication of noteworthy books by Iberoamerican authors -in their original language and in English translation-, and of learned works and textbooks.

Members of the Institute meet in Congresses every two years and are of two types: regular members who pay $\$ 6.00$ a year, except in Iheroamerica where the fee is $\$ 2.00$, and Patron Members who pay $\$ 10.00$ or more a year.

Institutions such as universities, colleges, and libraries may become subscribers (at $\$ 6.00$ a year or $\$ 2.00$ a year in Iberoamerica), or Subscribing Patrons (at a minimum of $\$ 10.00$ a year) without holding membership in the Institute.

Regular members and subscribers receive the forthcoming issues of the Revista Iberoamericana free, but Patrons (whether Members or Subscribers) receive, in addition, the forthcoming issues of all the publications of the Institute, such as the Clasicos de América, the Proceeding of the Congresses, etc., and their names will appear in the Revista Iberoamericana at the end of the year.

\section{N O T I C E}

We hope that you will become a member of the Institute, and if you cannot become one of its Patrons, we urge you to oblain a Patron Subscription for your school library, which then will receive the full cultural benefits of our publications.

Name of regular member or subscriber $(\$ 6.00) \ldots \ldots \ldots \ldots$

Name of Patron Member or Subscriber (\$10.00 minimum) ....

Address in full

Please make your checks payable to the Instituto.Internacional de Literatura Iberoamericana and mail your dues to Professor Saúl Sibirsky, Treasurer, University of Pittsburgh, Pittsburgh 13, Pennsylva. nia, U.S.A. All communications regarding the circulation and distribution of the publications of the Institute should also be addressed to Professor Sibirsky. 


\title{
SOCIOS Y SUSCRIPTORES
}

\author{
El instituto Internacional de Literatura Iberoamericana se \\ organizó en 1938 con el fin de incrementar el estudio de la Literatura \\ Iberoamericana $e$ intensificar las relaciones culturales entre todos los pue- \\ blos de América.
}

Con este fin, el Instituto publica la Revista Iberoamericana, por lo menos dos veces al año, y patrocina la publicación de obras notables de autores iberoamericanos - en el idioma original y en traducción inglesa-, y la de obras de erudición y textos de enseñanza.

Los socios del Instituto se reúnen en Congreso cada dos años, y son de dos categorías: el socio de número, cuya cuota anual es de seis dó. lares, excepto en Iberoamérica, donde es de sólo dos dólares, y el Socio Protector, cuya cuota es de diez dólares o más al año.

Las bibliotecas, colegios, universidades y demás instituciones que, sin ser socios, sí favorecen al Instituto, son de dos categorias: el suscriptor corriente, cuya cuota anual es de seis dólares y de sólo dos dólares en los países de Iberoamérica, y el Suscriptor Protector, cuya cuota es de dier dólares al año.

La Revista Iberoamericana se remite a los socios de número y a los suscriptores corrientes del Instituto, y tanto los Socios Protectores como los Suscriptores Protectores reciben, además de la Revista, las demás publicaciones que vayan saliendo, tales como los Clásicos de América y las Memorias, y sus nombres se publican en la Revista Iberoamericana al fin de cada año.

\section{N V I T A C I O N}

El Instituto invita cordialmente a quienes simpaticen con los fines que persigue, a que se hagan, ora socios, ora Protectores de él. Quienes asi lo apoyen deben enviar su cuota anual, por adelantado, en forma de giro postal o bancario pagadero al Instituto Internacional de Literatura Iberoamericana y por conducto del profesor Saúl Sibirsky, Secretario-Tesorero-CL-1617, University of Pittsburgh, Pittsburgh 13. Pennsylvania, U.S.A.-, que es la sinica persona encargada de la circulación y la distribución de las publicaciones del Instituto. 


\title{
Revista Iberoamericana
}

\author{
Organo del Instituto Internacional \\ de \\ Riteratura Iberoamericana
}

Vol. XXXIII

Enero-Junio, 1967

Núm. 63

\section{Publicación a cargo de:}

Director:

Alfredo A. Roggiano, C.L. I6r7, Universidad de Pittsburgh, Pittsburgh I3, Pennsylvania, U.S.A.

Secretario-Tesorero Interino:

Julio Matas, C.L. I6I7, Universidad de Pittsburgh, Pittsburgh I3, Pennsylvania, U.S.A.

Jefe de Canje:

Gutliermo Straub, C.L. I617, Universidad de Pittsburgh, Pittsburgh I3, Dennsylvania, U.S.A.

Jefe de Avisos:

Monique Lemaître-Nutini, C.L. I6r7, Universidad de Pittsburgh, Pittsburgh I3, Pennsylvania, U.S.A.

COMISIÓN EDITORIAL (I965-1967):

Enrique Anderson Imbert, Universidad de Harvard.

LuIS LEAL, Universidad de Illinois.

Wilson Martins, Universidad de New York.

ERnesto Mejía Sánchez, Universidad de México.

Carlos Solórzano, Universidad de México. 


\title{
INSTITUTO INTERNACIONAL DE LITERATURA IBEROAMERICANA
}

\author{
MESA DIRECTIVA
}

$(1965-1967)$

\section{PRESIDENTE}

Anibal Sánchez Reulet, Universidad de California, en Los Angeles.

VICEPRESIDENTES

Pedro Díaz Seijas, Vicepretsidente del Instituto Nacional de Cultura y Bellas Artes, Caracas, Venezuela.

Francisco Monterde, Presidente de la Academia Mexicana de la Lengua. Alfonso Rangel Guerra, Universided de Nuevo León, Monterrey, México.

DIRECTOR DE PUBLICACIONES

Alfredo 'A. Roggiano, Universidad de Pittsburgh.

\section{SECRETARIO EJECUTIVO-TEGORERO}

Saúl Sibirsky, Universidad de Pittsburgh.

(con licencia por dos años)

.SECRETARIO-TESORERO INTERINO

Julio Matas, Universidad de Pittsburgh.

COMISIÓN EDITORIAL

Enrique Anderson Imbert, Universidad de Harvard.

Luis Leal, Universidad de Illinois.

Wilson Martins, Universidad de New York.

Ernesto Mejía Sánchez, Universidad de México.

Carlos Solórzano, Universidaả de México. 


\section{REVISTA IBEROAMERICANA}

PROPOSITOS

Esta REvisTa aspira a constituir, gradualmente, usa vïtal representación de los valores espirituales de la creciente cultura iberoamericana.

Su director y asesores quieren hacer vivo el lema que cifra el ideal adoptado por nuestro Instituto: a LA FRATERNIDAD POR LA CUlTURA.

Reflejará en sus páginas una clara imagen de la literatura y del pensamiento de Iberoamérica.

\section{NITMAS EDI'TORIALES}

La REVISTA IBEROAMERICANA sólo publicará articulos aceptados por su director, quien será asesorado por la Comisión Editorial "Ad-hoc". Las ideas contenidas en lus artículos que se publiquen pertenecen al autor, quien será único responsable de las mismas.

Se recomienda que en los manuscritos de artículos, notas y reseñas presentados para su publicación se sigan las normas de "The MLA Style Sheet" publicado en PMLA, lxvi (1951).

\section{CANJE Y SUSCRIPCIONES}

Todo lc referente a CANJE y demás intercambio de publicaciones con casas editoras, instituciones o autores deberá hacerse por intermedio del Director-Editor, y a tal efecto se ruega dirigirse a: Alfredo A. Roggiano, Department of Romance Languages, University of Pittsburgh, Pittsburgh 13, Pennsylvania, U.S.A. Todo lo referente a sUSCRIPCIONEs, compras, órdenes de pago, etc., en que sea menester la intervención de la Tesorería, deberá hacerse por intermedio del Secretario Ejecutivo-Tesorero, y a tal efecto se ruega escribir a: SAÚl SibiRSky, Department of Romance Languages, University of Pittsburgh, Pittsburgh 13, Pennsyl. vania, U.S.A. 


\section{SOCIOS PROTECTORES}

\section{U.S.A.}

Abilene Christian College

College Library

Abilene, Texas 79601

David H. Allen

Department of Spanish

University of California

Davis, California 95616

J. B. Avalle-Arce

22 Paradise Rrad

Northampton, Mass. 01060

Gloria C. Astiazarán

Department of Foreing Lang

University of Nevada

Reno, Nevada 89507

Rodney V. Bodden

Dept. of Romance Languages

University of Washington

Seattle, Washington 98105

Florence Bonhard

83 Fremont Place

Los Angeles, California 9005

Wayne S. Bowen

Dept of Fureign Languages

Fresno State College

Fresno, California 93726

W. J. Bruner

Modern Language Department

William Jewell College

Liberty, Missouri

Luz Campana de Watts

5814 Summit View Ave.

Los Angeles 42, California

Professor Andrea I Pineda de Camps

Dept. of Romance \& German Languages

Wayne State University

Detroit, Michigan

Prof. José Ferrer-Canales

Box 811

Howard University

Washingtan $1, D$. C.
James A. Castañedo

Department of Classics

Rice University

Houston, Texas 77001

Homero Castillo

622 Sproul Hall

University of California

Dairs, California 95616

Prof. Boyd G. Carter

206 Pine Lane

Curbondale, Illinois

Othon Castillo

P.O. Box 5579

Metropolitan Station

Los Angeles, 55, California

Claremont College

The Honno!d Library

Periodicals Department

Claremont, California

H. Logan Ccbb

Dept. of Foreign Languages

Eastern Illinois University

Charleston, Illinois 61920

Colorade State College

Library

Creeley, Cclorado

Dra. Angela B. Dellepiane

Dept. of Romance Languages

City College

133rd at Convent Avenue

New York 31, N. Y. 10031

Pedro M. Duelo

1002 37th Street

San Pedro, California

Peter G. Earl

Dept. of Romance Languages

University of Pennsylvania

Philadelphia, Penna

The Library

Eastern New Mexico University

Bortales, New Mexico 88130
John E. Englekirk

Department of Spanish

University of California

Los Angeles 24, California

Felena Ferragut

St. John's University

Dept. of Modern Languages

Grand Central and Utopia Parkway

Jamaica 32, New York

Susana Redondo de Feldman

445 Riverside Drive

New York, N. Y. 10027

Dr. Esperanza FigueroaAmaral

Elmira College

Elmira, N. Y. 14901

Dr. Orlirio Fuentes

Dept. if Foreign Languages

Stcke Hall

Durham, New Hampshire

Elida A. Gigena

Watson 109

Nothern Illinois University

Dekalb, Illinois 60115

Prof. A. González

1617 C. L. University of Pittsburgh

Pittsburgh, Pa. 15213

Prof. A. A. Greene

Foreign Lang. Department

Trinity University

San Antonio, Texas 73212

Juana Amelia Hernández

Hood College

Frederick, Maryland 21702

Howard University

University Library

Serials Department

Washington, D. C. 20001 
Serials Department St. order

University of Illinois

University Library

Urbana, Illinois 61801

Library

Indiana University of Penna.

Indiana, Pennsylvania

State University of Iowa

Libraries

Serials Acquisition

Iowa City, Iowa 52240

Herbert Izzo

Foreign Lang. Dept.

San Jose State College

San Jose, California 95114

Didier T. Jaen

Spanish Department

University of California

Davis, California 95616

José Olivio Jiménez

392 Central Parkwest (20-J)

New York, N. Y. 10025

Sonja Karsen Dr.

Dept. of Modern Languages

Skudmore College

Saratoga Springs, N. $Y$. 12866

Robert B. Knox

Dept, of Foreign Languages

Washington State University

Pullman, Wash. 99163

Alyce G. De Kuchne

Dept. of Spanish \& Portuguese

University of California

Santa Barbara, California

Prof. Anthony Lamb

Western Illinois University

C-10 507 W. Adams Street

Macomb, Illinois 61455

Myron E. Lichtblau

Hall of Languages

Syracuse University

Syracuse, N. Y.

Carios Lozano

Dept. of Romance Languages

Sanint Mary's College

Saint Mary's College, Calif
Prof. Wilson Martins

1 Washington Square Village

Apt. B-8

New York, N. Y. 10012

María Maseda

44 Alice Drive

Manchester, Conn. 06040

Vera Maslow

315 W. 57th Street

The Towers

New York.19, N. Y.

Malcolm D. McLea

Texas Christian University

Fort Worth, Texas

Warren L. Meinhardt

Dept. of Span, Italian, \& Port.

University of Illinois

Urbara, Ill. 61803

Serge Mitrofaroff

715 S. Negley Ave.

Pittsburgh, Pa.

Edwin C. Munro

State Univ, of New York

135 Western Ave.

Albany, New York

Mildred A. Murphy

11284 Montana Ave.

Los Angeles, California 90049

Marshall R. Nason

Director, Language \& Area Center

University of New Mexico

Albuquerque, New Mexico

Graciela P. Nemes

6926 Pineway

Hyattsville, Maryland 20782

Mrs. Jean Nye

Dept. of Languages

Findlay College

Findlay, Obio

Otio Olivera

Department of Spanish Lang.

Tulane University

New Orięans, Lousiana 70118
Hextor H. Orjuela

Dept. of Spanish

U. S. C.

Los Angeles 7, California

Marta L. Pérez

6655 Marlow Drive

Jacksonville, Florida 32211

Library

Princeton University

Princeton, New Jersey

Prof. Manuel D. Ramírez

Dept. of Modern Foreign Lang.

University of Georgia

Athens, Georgia

Daniel R. Reedy

Dept, of Spanish \& Italian

University of Kentucky

Lexington, Kentucky

Carlos Ripoll

Department of Spanish

Queons College

Flushing 67, New York

Prof. James Willis Robi

Gecrge Washington University

Washington $6, \mathrm{D} . \mathrm{C}$.

Renato Rosaldo

Dept. of Romance Languages

University of Arizona

Tucson, Arizona

Dr. Ivan A: Schulman

Washington 'University

Department of Spanish

St. Louis Missouri 63130

Lawrence A. Sharpe

Asst. Prof. Spanish \& Port.

University of North Carolina

Chapel Hill, North Carolina

Raymond D. Souza

1409 W. 22nd Street

Lawrence, Kansas 6604

Douglas B. Swett

Chairman, Dept. of Romance

Languages \& World Litera. ture

Principia College

Elsah, Ill. 
Martin C. Taylor

University of Michigan

2079 Frieme Bldg-Spanish Dept.

Ann Arbor, Michigan

Ferdina J. C. Tort

University of IllinoisChicago

Dept. of Spanish

Chicago, Ill. 60680

\section{Library}

Trinity College

Hatford, Corn.
A. Valbuena (Bricnes)

203 Nottingham road

Newark, Delaware 19711

Luis Valdespino

Central Washington State College

Ellensburg, Washington 98926

Washington University

University Library

Saint Louis, Mo. 63130

Rev. Marion R. Watts OFM

San Luis Rey College

San Luis Rey, California
Dr. Grace E. Weeks Sanford University Birmingham, Ala. 35209

Library

Wellesley College

Wellesley, Mass.

\section{Library}

Williams College

Williamstcwn, Mass.

Margarita Winikoff 709 Washington Drive Pittsburgh, Pa. 15229

\title{
PUERTO RICO
}

\author{
Antilian College \\ Biblisteca \\ Colegio Adventista Puerto- \\ rriquez \\ P.O. Box 118 \\ Mayagüez, Puerto Rico
}

Dr. Giovanni Previtali

Universidad de Puerto Rico

Facultad de Artes y Ciencias

Mayagüez, Puerto Rico 00709 